\title{
Transjugular Intrahepatic Portosystemic Shunts for Hepatorenal Syndrome: TIPping the Scales in Whose Favor?
}

\author{
M. Asim Khokhar ${ }^{1}$. James O'Beirne ${ }^{1,2}$
}

Published online: 29 May 2020

(c) Springer Science+Business Media, LLC, part of Springer Nature 2020

\author{
Abbreviations \\ TIPS Transjugular intrahepatic portosystemic shunt \\ HRS Hepatorenal syndrome \\ LT Liver transplant \\ SIRS Systemic inflammatory response syndrome \\ HE Hepatic encephalopathy
}

Hepatorenal syndrome (HRS) is a life-threatening though potentially reversible complication of liver cirrhosis associated with high mortality. HRS usually occurs after a precipitating event such as bacterial infection that triggers severe circulatory dysfunction, inadequate cardiac output, and a proinflammatory state that substantially reduces renal perfusion [1]. Though the most successful treatment is liver transplantation (LT), this option is not applicable to the vast majority of HRS patients due to a lack of available organs and/or patient factors affecting candidacy. Hence, there is much interest in alternate therapies which could either reverse HRS or bridge a patient to successful LT.

Traditionally, the development of HRS has been based on the peripheral arterial vasodilation theory whereby splanchnic and peripheral vasodilation activated endogenous vasoconstrictors, progressing to renal vasoconstriction and ultimately HRS [2]. Accordingly, the application of vasoconstrictor therapy with volume expansion to address these circulatory changes has been adopted as rational treatment for the condition. Of the available therapies, the combination of octreotide, midodrine, and albumin is recommended in the USA, whereas the combination of terlipressin and

James O’Beirne

James.OBeirne@health.qld.gov.au; drjobeirne@gmail.com

1 Department of Gastroenterology and Hepatology, Sunshine Coast University Hospital, 6 Doherty St, Birtinya, QLD, Australia

2 Faculty of Science, Health, Education and Engineering, University of the Sunshine Coast (USC), 90 Sippy Downs Dr, Sippy Downs, QLD, Australia albumin has gained wider acceptance worldwide and is recommended by European guidelines. Encouragingly, a trend toward reduced inpatient mortality and improved survival in HRS has been observed over last two decades according to the National Inpatient Database-based retrospective cohort studies [3,4]. Nevertheless, in a recent meta-analysis of randomized controlled trials of different vasoconstrictor therapies, the pooled survival rate was only $23.8-37.7 \%$ despite a HRS reversal rate of $42.8 \%$. Notably, there was no improvement in outcomes over the decade of study [5].

Clearly, currently available pharmacological therapies reveal an unmet need in the treatment of HRS. Given that the pathogenesis of circulatory dysfunction in HRS is dependent on the presence of portal hypertension, it is perhaps unsurprising that TIPS has been explored for this indication. Despite some encouraging case reports and pilot studies reporting reversal of HRS and prolonged survival, the lack of controlled data and difficulties in providing TIPS to these very sick patients has meant that TIPS has not gained popularity for this indication and is considered experimental [6]. In those patients where currently available pharmacotherapies are unsuccessful, the otherwise poor outcome mandates that the role of other therapies such as TIPS is explored.

In this issue of Digestive Diseases and Sciences, Charilaou et al. [7] have performed a retrospective analysis of the National Inpatient Sample database from 2005 to 2014 using ICD-9-CM codes in order to identify patients with cirrhosis and HRS who received treatment with TIPS, comparing the outcome of these patients with those who received dialysis or no TIPS, finding that TIPS is associated with reduced inpatient mortality. The sample size is large, comprising $4,840,843$ patients with a diagnosis of cirrhosis of whom 79,354 had HRS recorded as a principal diagnosis. TIPS was performed in 918 of these patients and 10,379 received dialysis. The authors excluded patients with a diagnosis of variceal bleeding. The overall inpatient mortality was $18 \%$ in TIPS and $48 \%$ in dialysis-only cases $(n=10,379 ; 13 \%)$. Comparing outcomes, TIPS only patients were $57 \%$ less likely to die as inpatient compared to non-TIPS patients 
$[(\mathrm{aOR})=0.43,95 \%$ CI $0.30-0.63 ; p<0.001)$, whereas compared to the dialysis-only group, TIPS patients had a $69 \%$ less odds of dying as inpatients [contrast $(\mathrm{aOR})=0.31$, $95 \%$ CI $0.20-0.46 ; p<0.001$ ). This is an interesting finding that supports previous observational data that have suggested that TIPS can be applied successfully in well-selected patients with HRS, especially in the presence of preserved liver function [8]. Generic to studies utilizing administrative databases is that while the sample sizes are large, there is a lack of granularity regarding the clinical details of the patients involved to the point that it is difficult to draw conclusions from the data as to which patients may benefit.

For instance, in the current study, the biochemical data that would facilitate the direct comparison of renal and liver function between groups are unavailable. This limitation is acknowledged by the authors; in an attempt to mitigate selection bias, repeated analyses of the data to maximize the matching for several, but not all, variables between the analyzed groups were performed. For example, to exclude the possibility that the survival advantage observed was biased toward patients undergoing TIPS for refractory ascites, the authors found that the positive effect of TIPS on inpatient mortality were preserved with or without inclusion of ascites as a co-variable.

An interesting observation is the relative lack of mortality benefit among female patients, very similar to another recent study using the same sample and similar methodology which found no mortality advantage in females receiving TIPS [9]. This is an intriguing finding that deserves explanation. One possibility is the fact that females have a lower glomerular filtration rate (GFR) for a given serum creatinine compared to men. Serum creatinine therefore overestimates renal function in females and by extension underestimates liver disease severity according to MELD [10]. It is conceivable that this systematic bias could have led to the inclusion of an inherently sicker group of female patients.

Several differences at baseline between the TIPS, nonTIPS, and dialysis only groups were evident, reflecting the well-known contraindications to TIPS insertion such as septic shock, hepatocellular carcinoma, congestive heart failure, and pulmonary hypertension, suggesting a degree of appropriate selection. Somewhat surprisingly, the diagnosis of hepatic encephalopathy (HE) was present in 38.8-42.9\% of patients, not significantly different between the groups. A major limitation of the NIS database is the inability to discern if complications are present on admission or develop during admission. Given that overt HE would be considered a contraindication to TIPS insertion by many, it is tempting to speculate that the HE observed in the TIPS group is likely to be TIPS induced. If so, this rate is equivalent to that reported in other TIPS studies and perhaps explains why the length-of-stay in TIPS patients is significantly longer than in the non-TIPS and dialysis groups.
Though the observations made in the study are potentially important and interesting, they do not appear to be robust enough to change clinical practice regarding the applicability of TIPS in type-1 HRS. EASL guidelines strongly recommend terlipressin plus albumin as first-line treatment of type 1 HRS [11] based on available evidence from RCTs. The evidence-base supporting terlipressin plus albumin has expanded considerably; the recent results from the phase III CONFIRM trial $(n=300)$ demonstrating benefit of terlipressin plus albumin over placebo plus albumin are very encouraging, supporting its benefit in treating HRS even in very sick patients with high MELD scores and features of SIRS [12].

Therefore, it appears that terlipressin plus albumin is likely to become treatment of choice for HRS as a bridge to transplant, until and unless carefully designed randomized controlled trials with strict inclusion and exclusion criteria guided by available evidence evaluate the utility of TIPS for this particular indication.

Funding None.

\section{Compliance with Ethical Standards}

Conflict of interest The authors certify that they have no affiliations with or involvement in any organization or entity with any financial interest, or non-financial interest in the subject matter or materials discussed in this manuscript.

\section{References}

1. Angeli P, Garcia-Tsao G, Nadim MK, Parikh CR. News in pathophysiology, definition and classification of hepatorenal syndrome: a step beyond the international club of ascites (ICA) consensus document. J Hepatol. 2019;71:811-822.

2. Schrier RW, Shchekochikhin D, Gines P. Renal failure in cirrhosis: prerenal azotemia, hepatorenal syndrome and acute tubular necrosis. Nephrol Dial Transplant Off Publ Eur Dial Transpl Assoc Eur Ren Assoc. 2012;27:2625-2628.

3. Gopalakrishnan Ravikumar NP, Kailasam K, Ravichandar M, Chhatrala H. Hepatorenal syndrome: 2000-2014 inpatient hospitalization and mortality trends 2017.

4. Suneja M, Tang F, Cavanaugh JE, Polgreen LA, Polgreen PM. Population based trends in the incidence of hospital admission for the diagnosis of hepatorenal syndrome: 1998-2011. Int J Nephrol. 2016;2016:8419719.

5. Thomson MJ, Taylor A, Sharma P, Lok AS, Tapper EB. Limited progress in hepatorenal syndrome (HRS) reversal and survival 2002-2018: a systematic review and meta-analysis. Dig Dis Sci. 2019. https://doi.org/10.1007/s10620-019-05858-2.

6. Tripathi D, Stanley AJ, Hayes PC, et al. Transjugular intrahepatic portosystemic stent-shunt in the management of portal hypertension. Gut. 2020. https://doi.org/10.1136/gutjnl-2019-320221.

7. Charilaou P, Devani K, Petrosyan R, Reddy C, Pyrsopoulos N. Inpatient mortality benefit with transjugular intrahepatic portosystemic shunt for hospitalized hepatorenal syndrome patients. 
Dig Dis Sci. (Epub ahead of print). https://doi.org/10.1007/s1062 0-020-06136-2.

8. Song T, Rossle M, He F, Liu F, Guo X, Qi X. Transjugular intrahepatic portosystemic shunt for hepatorenal syndrome: a systematic review and meta-analysis. Dig Liv Dis Off J Ital Soc Gastroenterol Ital Assoc Study Liv. 2018;50:323-330.

9. Trivedi PS, Brown MA, Rochon PJ, Ryu RK, Johnson DT. Gender disparity in inpatient mortality after transjugular intrahepatic portosystemic shunt creation in patients admitted with hepatorenal syndrome: a nationwide study. $\mathrm{J} \mathrm{Am} \mathrm{Coll} \mathrm{Radiol.}$ 2020;17:231-237.

10. Cholongitas E, Marelli L, Kerry A, et al. Female liver transplant recipients with the same GFR as male recipients have lower MELD scores: a systematic bias. Am J Transplant Off J Am Soc Transplant Am Soc Transpl Surg. 2007;7:685-692.
11. European Association for the Study of the Liver. EASL clinical practice guidelines for the management of patients with decompensated cirrhosis. J Hepatol. 2018;69:406-460.

12. Wong F, Curry M, Reddy K, et al. The confirm study: a North American randomized controlled trial (RCT) of terlipressin plus albumin for the treatment of hepatorenal syndrome type 1 (Hrs-1). Hepatology. 2019;70:1480A-1A.

Publisher's Note Springer Nature remains neutral with regard to jurisdictional claims in published maps and institutional affiliations. 